\title{
Enhanced anti-tumor immune responses and delay of tumor development in human epidermal growth factor receptor 2 mice immunized with an immunostimulatory peptide in poly( $D$, L-lactic-Co- glycolic) acid nanoparticles
}

\author{
Diahnn F Campbell ${ }^{1}$, Rebecca Saenz ${ }^{1}$, lla S Bharati ${ }^{1}$, Daniel Seible ${ }^{1}$, Liangfang Zhang ${ }^{2}$, Sadik Esener ${ }^{3}$, \\ Bradley Messmer ${ }^{1 *}$, Marie Larsson ${ }^{4}$ and Davorka Messmer ${ }^{1,5}$
}

\begin{abstract}
Introduction: Cancer vaccines have the potential to induce curative anti-tumor immune responses and better adjuvants may improve vaccine efficacy. We have previously shown that Hp91, a peptide derived from the B box domain in high-mobility group box protein 1 (HMGB1), acts as a potent immune adjuvant.

Method: In this study, Hp91 was tested as part of a therapeutic vaccine against human epidermal growth factor receptor 2 (HER2)-positive breast cancer.

Results: Free peptide did not significantly augment immune responses but, when delivered in poly(D,L-lactic-coglycolic) acid nanoparticles (PLGA-NPs), robust activation of dendritic cells (DCs) and increased activation of HER2specific T cells was observed in vitro. Vaccination of HER2/neu transgenic mice, a mouse breast cancer model that closely mimics the immune modulation and tolerance in some breast cancer patients, with Hp91-loaded PLGA-NPs enhanced the activation of HER2-specific cytotoxic T lymphocyte $(\mathrm{CTL})$ responses, delayed tumor development, and prolonged survival.
\end{abstract}

Conclusions: Taken together these findings demonstrate that the delivery of the immunostimulatory peptide Hp91 inside PLGA-NPs enhances the potency of the peptide and efficacy of a breast cancer vaccine.

\section{Introduction}

Vaccines are a promising approach to prevent or cure cancer $[1,2]$ but generally require a tumor antigen and an immune-stimulatory adjuvant. Breast cancers that express the human epidermal growth factor receptor 2 (HER2) have been treated with some success by immunotherapies that target that antigen [3]. Vaccines can be potentiated by their method of administration and formulation. For example, nanoparticles (NPs) can protect sensitive/and or unstable antigens such as peptides from degradation and potentially increase the immune

\footnotetext{
* Correspondence: bmessmer@ucsd.edu

1 Moores UCSD Cancer Center, University of California San Diego, 3855 Health Sciences Drive, La Jolla, CA 92093-0815, USA

Full list of author information is available at the end of the article
}

response to vaccines. It has been shown that encapsulation of antigen into biodegradable spheres leads to enhanced humoral and cellular immune responses [4,5]. Poly(D,L-lactic-co-glycolic) acid nanoparticles (PLGANPs) have been used to deliver the cancer-associated antigen MUC1,5,6 as well as tetanus toxoid to enhance immune responses [6]. PLGA is a biodegradable and biocompatible polymer $[7,8]$ with good stability in the gastrointestinal tract [9] and is used for numerous in vivo applications $[10,11]$. NPs also have the advantage that, by using different polymer compositions, one can control the release of cargo allowing for antigen depot formation at the injection site. These manipulations might provide enabling technologies to the vaccine as well as drug development field. 
Dendritic cells (DCs) are the most potent antigenpresenting cells and are critical for the initiation of adaptive immune responses. Vaccines need to stimulate DCs to induce potent immune responses. DCs must receive a maturation signal to present antigen, upregulate costimulatory and adhesion molecules, and become potent activators of $\mathrm{T}$ cells [12]. The immunostimulatory peptide Hp91, which is derived from the endogenous protein high-mobility group box protein 1 (HMGB1), activates DCs [13] and primes antigen-specific cytotoxic T lymphocyte (CTL) responses in vitro [13] and in vivo [14]. Hp91 packaged inside of PLGA-NPs is more potent in activating DCs as compared to free peptide [15]. In our previous study, the PLGA-NPs were synthesized using an emulsion method yielding non-homogeneous particles. In the current study, we used a precipitation method that yields homogeneous NPs, to package Hp91 inside PLGA-NPs. We evaluated the extent to which Hp91-PLGA-NPs protect against breast cancer using a HER2 breast cancer mouse model [16]. Our results demonstrate that the delivery of the immunostimulatory peptide $\mathrm{Hp} 91$ inside the PLGA-NPs enhances the efficacy of this breast cancer vaccine.

\section{Materials and methods Peptides}

The adjuvant peptide Hp91 (DPNAPKRPPSAFFLFCSE) and MHC class I $\left(\mathrm{H} 2-\mathrm{D}^{\mathrm{q}}\right)$-restricted rat HER-2/neuderived peptide (PDSLRDLSVF) were both purchased from CPC Scientific (San Jose, CA, USA). The Hp91 peptide was synthesized with an $\mathrm{N}$-terminal biotin and dissolved in RPMI for in vitro studies and phosphatebuffered saline (PBS) for immunizations. The HER2 peptide was dissolved in 3\% dimethyl sulfoxide (DMSO)/PBS. Peptides were routinely synthesized with greater than $95 \%$ purity.

\section{Animals}

FVB.N/neu-tg mice were derived from in-house breeding stocks at the University of California, San Diego (UCSD) Moores Cancer Center animal facility. All animal studies were approved by the Institutional Animal Care and Use Committee of the University of California, San Diego and performed in accordance with the institutional guidelines.

\section{Synthesis of peptide-loaded lipid-polymer hybrid nanoparticles}

Ester-terminated poly-lactic-co-glycolic acid, or PLGA (50:50, $0.82 \mathrm{dl} / \mathrm{g}$ IV, DURECT Corporation, Cupertino, CA, USA) was dissolved at $1 \mathrm{mg} / \mathrm{ml}$ in dimethylformamide (DMF). Hp91 was also dissolved in DMF with the PLGA at concentrations of 1 to $5 \mathrm{mg} / \mathrm{ml}$. Lecithin (molecular weight (MW) $330 \mathrm{Da}$, Alfa Aesar, Ward Hill,
MA, USA) and 1,2-distearoyl-sn-glycero-3-phosphoethanolamine-N-(carboxy(polyethylene glycol)2000) (ammonium salt) (DSPE-PEG-Carboxy, MW 2,849.54 Da, Avanti Polar Lipids, Alabaster, AL, USA) were dissolved together in $2 \mathrm{ml}$ of $4 \%$ ethanol per mg PLGA to be used at a ratio of $9 \%$ of total PLGA weight for lecithin and $52 \%$ of total PLGA weight for DSPE-PEG-Carboxy. All stock solutions were made using sterile solvents or endotoxin-free water. The aqueous lipid mixture was heated to $68^{\circ} \mathrm{C}$ while stirring for $3 \mathrm{~min}$. The PLGA-peptide solution was added dropwise to the heated lipid solution while stirring. The solution was then vortexed at 3,000 RPM for three minutes. An additional $1 \mathrm{ml}$ of water per mg of PLGA used was added dropwise to the NP solution while stirring. The NP solution was stirred without cap for $2 \mathrm{~h}$ to allow solvent evaporation. The particles were then washed three times using Amicon Ultra centrifugal filter devices by EMD Millipore (Billerica, MA, USA) with $100 \mathrm{Kd}$ cutoff. Particles were suspended in 10\% sucrose and flash frozen for later use.

\section{Characterization of lipid-polymer polylactic-co-glycolic acid hybrid nanoparticles (PLGA-NPs)}

The NP formation was analyzed for particle size by dynamic light scattering (DLS) using a zetasizer (Zetasizer Nano ZS, Malvern Instruments Ltd, Malvern, UK). To quantify the amount of peptide loaded into the hybrid NPs, the NPs were dissolved in DMF for 30 min under constant shaking at room temperature and peptide content was quantified by high-performance liquid chromatography (HPLC) (column: Waters Delta-Pak C18 5 microns, Waters Corporation, Milford, MA, USA) at $211 \mathrm{~nm}$ in comparison to a Hp91 peptide standard curve. To measure the release rate of the peptide from the NPs, $100 \mu \mathrm{L}$ of Hp91-loaded NP solution was added to microdialysis cassettes with a MW cutoff of 10,000 and dialyzed against $1 \mathrm{~L}$ of PBS buffer at $\mathrm{pH} 7.4$ or potassium hydrogen phthalate buffer at $\mathrm{pH} 5$. At each time point, two samples for each buffer condition were recovered from the microdialysis cassettes, and the volumes were brought up to $125 \mu \mathrm{L}$ to keep all volumes constant. To each sample, $125 \mu \mathrm{L}$ of DMF was added to dissolve the NPs and release the remaining Hp91 peptide. The samples were shaken for $60 \mathrm{~min}$, and then the total amount of $\mathrm{Hp} 91$ in each sample was quantified using HPLC. The amounts were normalized against the starting concentration of peptide before dialysis, which was set at $100 \%$ to calculate the percentage released.

\section{Generation of mouse bone marrow-derived DCs}

Bone marrow-derived dendritic cells (BM-DCs) were prepared from HER-2/neu transgenic mice $\left(\mathrm{H}-2^{\mathrm{q}}\right)$, as described by Inaba et al. [17] with minor modifications. Briefly, single bone marrow cell suspensions were obtained 
from femurs and tibias, depleted of lymphocytes, granulocytes, and Ia + cells using a mixture of monoclonal antibodies (mAbs; anti-CD4, anti-CD8, anti-B220/CD45R, and anti-Ia) for $45 \mathrm{~min}$ on ice, followed by incubation with low-toxicity rabbit complement for $30 \mathrm{~min}$ at $37^{\circ} \mathrm{C}$. Cells were resuspended at a concentration of $10^{6}$ cells/ $\mathrm{mL}$ in medium supplemented with recombinant murine granulocyte-macrophage colony-stimulating factor (GMCSF) $(10 \mathrm{ng} / \mathrm{mL})$. Fresh medium $(5 \% \mathrm{vol} / \mathrm{vol}$ fetal calf serum (FBS)-RPMI) containing GM-CSF was added on day 2 and 4 of culture. On day 6, cells were collected for the experiments.

\section{Antigen presentation assays}

Immature BM-DCs $\left(10^{5}\right)$ were stimulated with media alone, similar amounts of Hp91 free peptide, NP-encapsulated Hp91, or $10 \mathrm{ng} / \mathrm{mL}$ lipopolysaccharide (LPS) (SigmaAldrich, St Louis, MO, USA). Forty-eight hours after activation, the cells were incubated with $100 \mathrm{ng} / \mathrm{mL}$ HER2 peptide for $1 \mathrm{~h}$ at $37^{\circ} \mathrm{C}$. The cells were then washed twice to remove excess peptide and plated with HER2-specific CTL clones (kindly provided to us by Professor E. Jaffee (John Hopkins Medical Institute) at a $10^{3}: 10^{4} \mathrm{DC}$ to $\mathrm{T}$ cell ratio in wells of a nitrocellulose bottom enzyme-linked immunospot (ELISPOT) plate (EMD Millipore) that had been previously coated overnight with $5 \mu \mathrm{g} / \mathrm{mL}$ monoclonal anti-mouse interferon gamma (IFN- $\gamma$ ) antibody (Mabtech, Stockholm, Sweden). After $18 \mathrm{~h}$, the ELISPOT plates were developed using $1 \mu \mathrm{g} / \mathrm{ml}$ biotinylated anti-mouse IFN- $\gamma$ antibody (Mabtech), Streptavidin-horseradish peroxidase (HRP) (Mabtech), and 3,3',5,5'-Tetramethylbenzidine (TMB) substrate (Mabtech). The plate was scanned and the spots were counted using an automated ELISpot Reader System (CTL ImmunoSpot, Shaker Heights, OH, USA).

\section{Immunizations and spleen cell preparation}

The HER-2/neu peptide antigen was co-administered subcutaneously with either PBS, soluble Hp91, or NPencapsulated Hp91 on the right flank. Spleens were collected 8 days after the final immunization. Single cell suspensions of splenocytes were prepared by mechanical disruption and separation through a 70- $\mu \mathrm{m}$ nylon cell strainer (BD Biosciences, Franklin Lakes, NJ, USA). Red blood cells were lysed using ammonium chloride buffer (Roche Diagnostics, Indianapolis, IN, USA) and the splenocytes were subsequently resuspended in complete medium (RPMI 1640 with 10\% FBS, L-glutamine, penicillin, streptomycin, and HEPES) supplemented with $20 \mathrm{U} / \mathrm{mL}$ of recombinant mouse interleukin (IL)2) (R\&D Systems, Minneapolis, MN, USA) and $10 \mu \mathrm{g} /$ $\mathrm{mL}$ of PDSLRDLSVF peptide for expansion. Splenocytes were expanded for 5 days prior to use in ELISPOT experiments.

\section{Enzyme-linked immunospot assay}

The expanded splenocytes were collected and washed twice before being plated in duplicate $10^{6}$ cells to wells of an ELISPOT plate that had been previously coated overnight with $5 \mu \mathrm{g} / \mathrm{mL}$ monoclonal anti-mouse IFN- $\gamma$ antibody. Splenocytes were cultured overnight at $37^{\circ} \mathrm{C}$ with $2.5 \mu \mathrm{g} / \mathrm{mL}$ HER2 peptide, $5 \mu \mathrm{g} / \mathrm{ml}$ concavalin A (Sigma-Aldrich) as positive control or left unstimulated (medium only). After $18 \mathrm{~h}$, ELISPOT plates were developed using $1 \mu \mathrm{g} / \mathrm{ml}$ biotinylated anti-mouse IFN- $\gamma$ antibody, streptavidin-HRP, and TMB substrate. The plate was scanned and the spots were counted using an automated ELISpot Reader System.

\section{Tumor prevention experiments}

Female HER-2/neu mice, 8 weeks of age, were immunized with $5 \mu \mathrm{g}$ of HER2 antigen mixed with either PBS only, $25 \mu \mathrm{g}$ of $\mathrm{Hp} 91$ free peptide, or $25 \mu \mathrm{g}$ of Hp91 delivered in PLGA-NPs. Mice received their first boost 2 weeks post-prime, and a second boost 1 month thereafter. All injections were performed subcutaneously on the right flank of the mice. The incidence and growth of tumors were evaluated twice a week by measuring palpable tumors, defined as tumors with diameters that exceed $3 \mathrm{~mm}$, with calipers in two perpendicular diameters. Calipers were used to measure tumor length and width and the volume was calculated as volume $\left(\mathrm{mm}^{3}\right)=(\text { width })^{2} \times$ length $/ 2$. All mice bearing tumor masses exceeding $1.5 \mathrm{~cm}$ mean diameter were sacrificed.

\section{Statistical analysis}

Data represented are mean \pm standard error of the mean (SEM). Data were analyzed for statistical significance using unpaired Student's $t$ test. Statistical analyses were done using GraphPad software version 5.01 for Windows (GraphPad Software, San Diego, CA, USA). A $P$ value $<0.05$ was considered statistically significant for these analyses.

\section{Results}

Delivery of Hp91 in PLGA-NPs increases its immunostimulatory capacity in vitro

We have previously shown that delivery of the immunostimulatory peptide Hp91 in PLGA-NPs leads to increased activation of both mouse and human DCs in vitro [14]. Those NPs were made using an emulsion method, which results in NPs that are very heterogeneous in size [15]. To develop a vaccine that can be taken into the clinic, it is critical to synthesize homogenous particles. Therefore, we have evaluated a nanoprecipitation method to incorporate Hp91. This method generates very homogeneous PLGA-NPs (Figure S1B in Additional file 1). $\mathrm{Hp} 91$ was efficiently released at $\mathrm{pH} 5$ and faster release was observed at pH 7.4 (Figure S1A in Additional file 1). 
To examine whether Hp91 maintains its DC-stimulatory properties $[14,18]$ when packaged inside NPs, immature human monocyte-derived DCs were exposed to increasing doses of either free Hp91 peptide or Hp91-loaded NPs. Hp91 incorporated in PLGA-NPs caused increased activation of DCs in vitro as measured by increased secretion of IL-6, as compared to free peptide (Figure S1C in Additional file 1). Next, we investigated whether the encapsulation of Hp91 could enhance HER2-specific T cell responses. BM-DCs from HER2/neu transgenic mice were assessed for their ability to induce antigen-specific $\mathrm{T}$ cell responses as measured by IFN- $\gamma$ secretion in an ELISPOT assay. DCs were exposed to media, free Hp91 peptide or encapsulated in PLGA-NPs or LPS for $48 \mathrm{~h}$. The DCs were then washed and pulsed with HER-2/neu peptide and tested for their ability to activate antigenspecific CTL responses using a HER-2/neu-specific CTL line. Hp91 loaded inside PLGA-NPs was significantly more potent in activating DCs, generating nearly a 4fold increase in HER-2/neu-specific CTL responses when compared to Hp91 delivered in its free form (Figure 1, $P<0.005)$.

Hp91 delivered in PLGA-NPs increases immune responses to the HER2/neu antigen in vivo

The transgenic HER2/neu mouse model was chosen as it had been shown to closely mimic the immune modulation

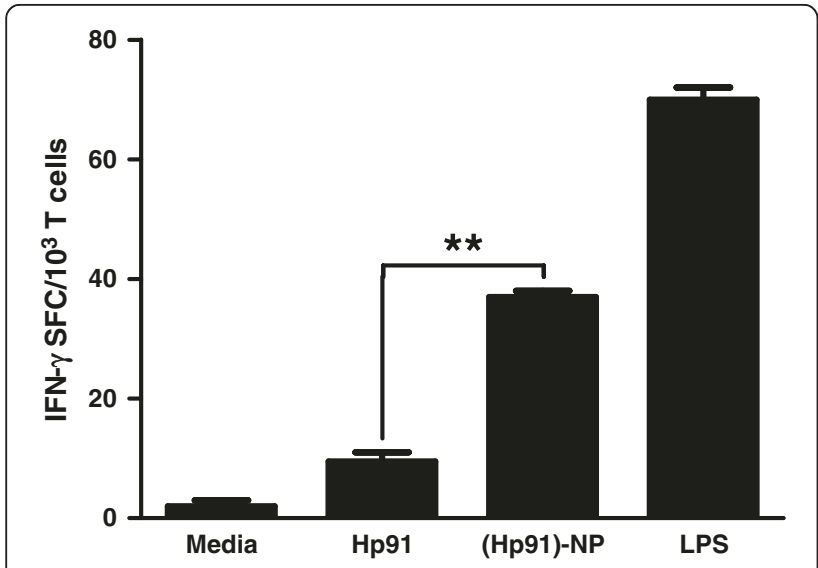

Figure 1 Delivery of Hp91 in nanoparticles to BM-DCs increases HER2-specific CTL responses in vitro. Immature mouse BM-DCs $\left(10^{5}\right)$ were exposed to media, $55 \mathrm{\mu g} / \mathrm{ml}$ of free $\mathrm{Hp} 91$ peptide or the same amount delivered inside PLGA-NPs, or lipopolysaccharide (LPS;

$10 \mathrm{ng} / \mathrm{mL}$ ). After 2 days the cells were collected, washed, and pulsed with HER2 peptide $(100 \mathrm{ng} / \mathrm{mL})$ for $1 \mathrm{~h}$ prior to the overnight co-culture with the HER-2/neu-specific CTL line in an ELISPOT assay.

The number of IFN- $\gamma$ spot-forming cells is shown as means ( \pm standard error of the mean (SEM)) of two independent experiments. ${ }^{* *}$ Indicates statistical significance $P<0.005$. BM-DCs, bone marrow-derived dendritic cells; CTL, cytotoxic T lymphocyte; ELISPOT, enzyme-linked immunospot; HER2, human epidermal growth factor receptor 2; IFN- $\gamma$, interferon gamma; PLGA-NPS, poly(D,L-lactic-co-glycolic) acid nanoparticles. and tolerance described in some breast cancer patients [16]. We tested if Hp91 when delivered in PLGA-NPs would be sufficiently potent to break tolerance and induce potent CTL responses in vivo. HER2/neu transgenic mice were vaccinated at three different time points starting at 2 months of age with HER2 peptide mixed with free Hp91, Hp91-loaded PLGA-NPs, or PBS control. Splenocytes from immunized mice were analyzed for HER2specific CD8 $+\mathrm{T}$ cell responses by IFN $\gamma$ ELISPOT assay (Figure 2A). Although an increase in immune response was observed when free Hp91 was co-injected with the HER2 peptide antigen, it was not statistically significant. However, when mice were injected with a similar dose of Hp91 encapsulated inside PLGA-NPs together with HER2 peptide antigen, a significant increase in HER2-specific IFN- $\gamma$ spot-forming cells as compared to the control group was measured $(P<0.0001)$ (Figure $2 \mathrm{~A})$. In addition, encapsulation of Hp91 peptide inside PLGA-NPs also enhanced the immune response when compared to mice immunized with free Hp91 $(P=0.015)$ (Figure 2A). Next, we tested whether the free Hp91 peptide at higher doses could induce immune responses in the HER2 mice. The mice were immunized according to the schedule above, but with a 10-fold higher dose of $250 \mu \mathrm{g}$ of free Hp91 and $14 \mu \mathrm{g}$ of Hp91 packaged in PLGA-NPs (Figure 2B). Even at higher doses free Hp91 peptide did not induce significant CTL responses in this mouse model. However, when packaged inside of PLGA-NPs even at an 18-fold lower dose of Hp91 as compared to free peptide, induced a significant increase in CTL responses. This shows that when delivered in PLGA-NPs the immunostimulatory peptide Hp91 shows increased adjuvant potency in vivo.

\section{Immunization of HER2/neu mice with Hp91 loaded in PLGA-NPs delays tumor development and prolongs survival}

The transgenic HER2/neu mice spontaneously develop mammary tumors beginning at 4 months of age. Therefore, we sought to investigate if our nanoparticle vaccine could prevent or delay tumor development in these mice. Mice received subcutaneous (s.c.) injections of the different vaccine compositions at 2 months of age and were monitored for development of palpable tumors twice a week. Mice were sacrificed once tumor masses exceeded $1.5 \mathrm{~cm}$ mean diameter. By 8.5 months of age, all mice from the control HER2 peptide antigen only group had developed tumor masses. Although all mice vaccinated with free Hp91 together with free HER2 peptide developed tumors, the vaccine delayed tumor development by approximately 2 months in some mice in comparison to mice immunized with the HER2 peptide antigen only control group (Figure 3A). Immunization of mice with Hp91 packaged in PLGA-NPs mixed with free HER2 peptide delayed the measurable appearance of tumors by 

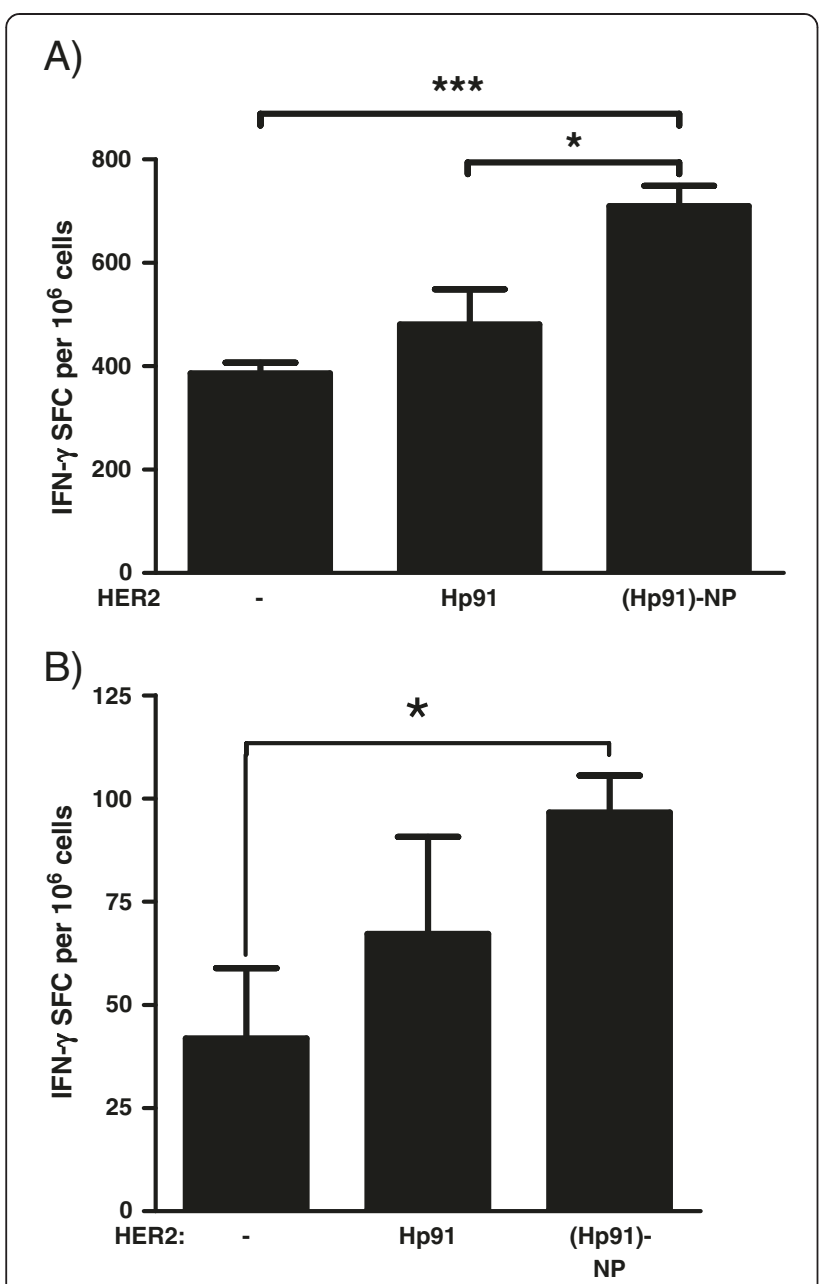

Figure 2 Immunization of HER2/neu transgenic mice with Hp91 in nanoparticles increases CTL responses. (A) HER2/neu transgenic mice at 2 months of age were immunized with $5 \mu \mathrm{g}$ HER2 peptide in PBS, or with $25 \mu \mathrm{g} \mathrm{Hp91}$ free peptide or the same amount of Hp91 packaged within PLGA-NPs. (B) A different group of HER2/neu transgenic mice was immunized at 4 months of age with $20 \mu \mathrm{g}$ HER2 free peptide or $4.5 \mu \mathrm{g}$ HER2 peptide inside PLGA-NP in PBS, alone or in combination with $250 \mu \mathrm{g} \mathrm{Hp} 91$ free peptide or $14 \mu \mathrm{g}$ Hp91 in PLGA-NPs. (A and B) Eight days after the final immunizations, mice were sacrificed and expanded splenocytes were cultured in the presence of HER2 peptide in an IFN- $\gamma$ ELISPOT assay. CTL responses were determined $18 \mathrm{~h}$ later by quantifying the number of IFN- $\gamma$ spotforming cells using an automated ELISPOT reader. Data shown represents the means ( \pm standard error of the mean (SEM)) for five mice per group. Indicates statistical significance $P<0.05$. CTL, cytotoxic T lymphocyte; ELISPOT, enzyme-linked immunospot; HER2, human epidermal growth factor receptor 2; IFN- $\gamma$, interferon gamma; PBS, phosphate-buffered saline; PLGA-NPs, poly(D,L-lactic-co-glycolic) acid nanoparticles.

an additional approximately 3 months compared to control mice and prolonged the overall survival (Figure 3B). These results indicate that the immunostimulatory peptide Hp91 when packaged inside of PLGA-NPs significantly enhances the anti-tumor response to free HER2 peptide in a prophylactic vaccine setting and yielded the longest survival (Figure 3B). Mice immunized with just the HER2 antigen alone were all sacrificed by approximately 10 months of age due to tumor mass. Increased immune responses correlated with protection from tumor development correlated.

\section{Discussion}

The activation of potent immune responses by vaccines or therapies is essential and not always achieved by traditional vaccines or therapies. The use of NPs to deliver adjuvant and antigen is now being thoroughly investigated. Tumor-associated antigens have been identified for a variety of cancers but so far very few clinical trials have shown great efficacy. This could have many reasons including immune suppression by the tumor microenvironment, poorly immunogenic antigens, limited $\mathrm{T}$ cell repertoire and so on. Another possibility could be deficiency in the vaccine delivery systems. The immune system generally responds to viruses and bacteria, which are particles and not isolated molecules and they contain the antigen in close proximity to the adjuvant. Various NPs are being explored as delivery tools for drugs, vaccine antigens and adjuvants. NPs are a delivery platform that can be tuned for different needs. The material used to make NPs can in itself be immunogenic, for instance poly $(\gamma$-glutamic acid) activates the immune system via TLR4/MyD88 NP [19]. Our goal was to use inert materials like PLGA-NPs as a neutral carrier, which can be loaded with immunogenic moieties, in this case the Hp91 peptide, to trigger desired immune responses. One reason for using non-stimulatory materials and loading them with adjuvant is that we believe understanding the mechanism behind material-induced immune responses is difficult, and using characterized adjuvants should be of advantage. The PLGA-NPs used in this study are biodegradable and biocompatible polymer10-13 that has been employed for numerous in vivo applications. We have not observed immune activation with the PLGA-NPs alone [16]. The PLGA-NPs used were $100 \mathrm{~nm}$ (range 30 to $200 \mathrm{~nm}$ ), a size which has been shown to be preferentially taken up by professional antigen-presenting cells (APCs) and are expected to enter DCs or Langerhans cells (LCs) when given transcutaneous or s.c..

We found that Hp91, when packaged inside of PLGANPs, activated both mouse and human DCs in vitro and induced increased CD8+ T cell responses as compared to free peptide. One possible explanation is that the delivery is more efficient, because the NPs are readily taken up by DCs and each NP will deliver many peptides, whereas free peptide will diffuse around the cells and the uptake is much less effective. Interestingly, the intensity of CTL responses seen in the immunized mice mirrored protection from tumor development. 
A

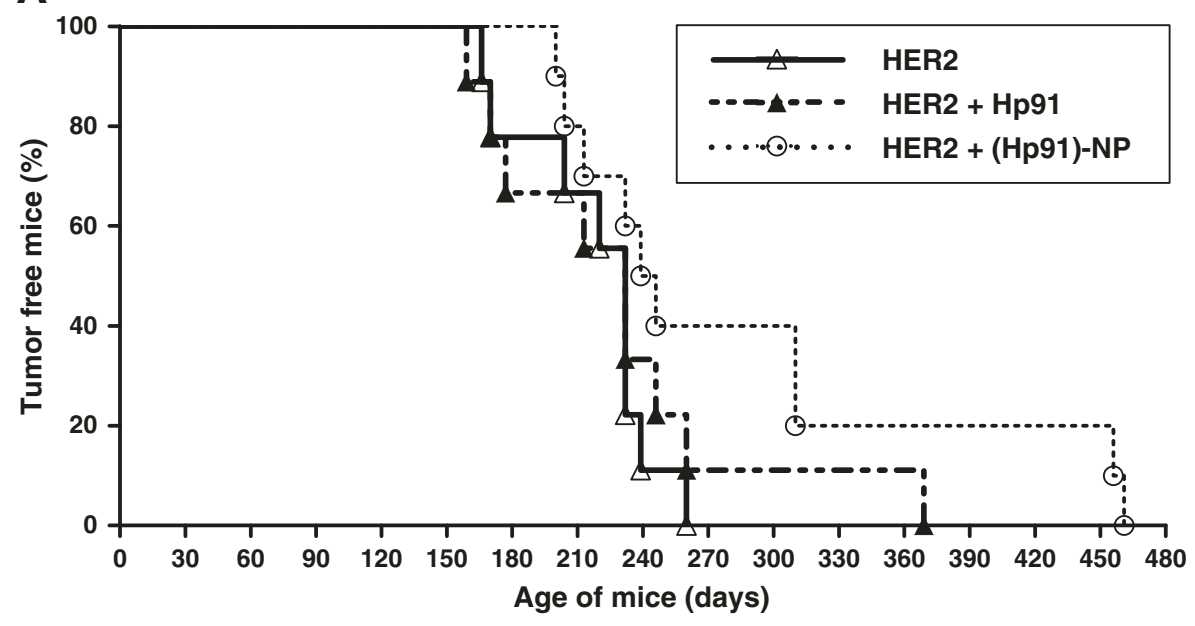

B

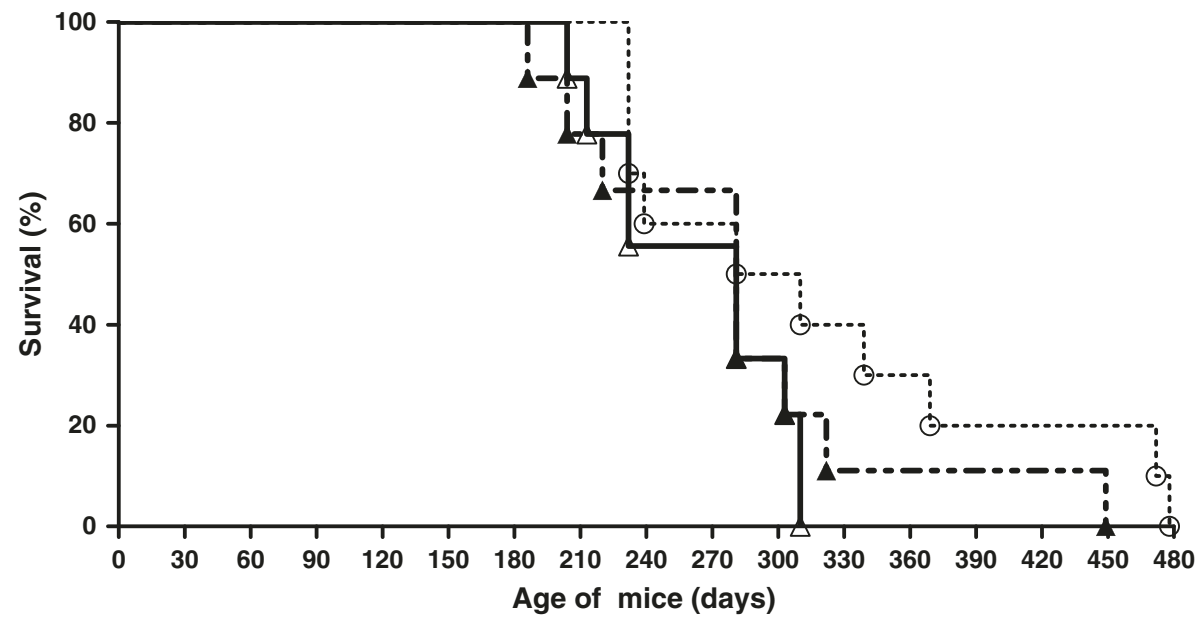

Figure 3 Immunization of HER2/neu-transgenic mice with Hp91 in nanoparticles delayed tumor development and prolonged survival. At 2 months of age, mice were immunized with $5 \mu \mathrm{g}$ HER2 peptide in PBS, $25 \mu \mathrm{g}$ of Hp91 free peptide, or $25 \mu \mathrm{g}$ Hp91 delivered in PLGA-NPs. After final immunization, mice were monitored twice a week for tumor growth and survival. (A) Percentages of tumor-free mice were calculated as cumulative number of mice with tumor and mice that were tumor free. (B) Mice were sacrificed once tumor masses exceeded $1.5 \mathrm{~cm}$ mean diameter. HER2, human epidermal growth factor receptor 2; PBS, phosphate-buffered saline; PLGA-NPs, poly(D,L-lactic-co-glycolic) acid nanoparticles.

\section{Conclusions}

Vaccination with HER2 peptide combined with Hp91loaded PLGA-NPs broke tolerance against the HER2 antigen in the HER2/neu transgenic mice and prolonged survival. This vaccine warrants further investigation for efficacy in other models as well as for safety. If proven safe in clinical trials, an interesting consideration would be to vaccinate patients with precancerous lesions as, for example, patients with HER2-positive ductal carcinoma in situ. These patients will likely have a fully functional immune system and should show good response to the vaccine whereas late-stage HER2-positive patients like any late-stage cancer patients will have an impaired immune responses and lower likelihood of success. Combinations with new therapies like anti-PD1 or anti-
CTLA4 antibodies might yield better success in latestage patients.

\section{Additional file}

Additional file 1: Figure S1. Characterization of PLGA-NPS. (A) Release of Hp91 peptide from PLGA-NPS and (B) PLGA-NP size was measured as previously described [18]. (C) Immature DCs were exposed to PLGA-NP-

loaded Hp91 or the equivalent amount of free peptide for $48 \mathrm{~h}$. Cell

culture supernatants were analyzed for IL-6 by ELISA.

\section{Abbreviations}

APCs: antigen-presenting cells; BM-DCs: bone marrow-derived dendritic cells; CTL: cytotoxic T lymphocyte; DCs: dendritic cells; DLS: dynamic light scattering; DMF: dimethylformamide; DMSO: dimethyl sulfoxide; DSPE-PEG-Carboxy: 1,2distearoyl-sn-glycero-3-phosphoethanolamine-N-(carboxy(polyethylene glycol) 2000); ELISPOT: enzyme-linked immunospot; FBS: fetal bovine serum; 
GM-CSF: granulocyte-macrophage colony-stimulating factor; HER2: human epidermal growth factor receptor 2; HMGB1: high-mobility group box protein 1; HPLC: high-performance liquid chromatography; HRP: horseradish peroxidase; IFNY: interferon gamma; IL: interleukin; LCs: Langerhans cells;

LPS: lipopolysaccharide; mAbs: monoclonal antibodies; MW: molecular weight; NP: nanoparticle; PBS: phosphate-buffered saline; PLGA: poly(D,L-lactic-co-glycolic) acid; PLGA-NPs: poly(D,L-lactic-co-glycolic) acid nanoparticles; s.c.: subcutaneous; TMB: 3,3',5,5'-Tetramethylbenzidine.

\section{Competing interests}

The authors declare that they have no competing interests.

\section{Authors' contributions}

DFC isolated DC and performed immune assays in vitro, synthesized nanoparticles, immunized mice and performed immune readouts after vaccination, plotted data and drafted the manuscript. RS helped with immunization, isolation of immune cells, and critical revision of the manuscript. ISB bred the mouse colony, helped with immunizations and was involved in drafting the manuscript. DS synthesized nanoparticles and was involved in drafting the manuscript. LZ designed nanoparticles and was involved in drafting the manuscript. SE helped with nanoparticle analysis and characterization and was involved in drafting the manuscript. BM helped with experimental design, wrote parts of the manuscript and was involved in critical revision of the manuscript. ML helped with experimental design and analysis, wrote parts of the manuscript and was involved in critical revision of the manuscript. DM conceived of the study, guided the project, designed experiments, analyzed data, supervised the team, drafted, and wrote the manuscript. All authors have given final approval of the version to be published and agree to be accountable for all aspects of the work in ensuring that questions related to the accuracy or integrity of any part of the work are appropriately investigated and resolved.

\section{Authors' information}

D.F. Campbell was formerly Futalan.

\section{Acknowledgements}

This work was supported by the US Army Medical Research and Material Command under Agreement No. W81XWH-07-1-0412 (to D.M), 5U54CA119335 from the National Institutes of Health/NCl (to S.E.). We wish to thank Esther Avery for advice on mouse breeding.

\author{
Author details \\ ${ }^{1}$ Moores UCSD Cancer Center, University of California San Diego, 3855 Health \\ Sciences Drive, La Jolla, CA 92093-0815, USA. ${ }^{2}$ Department of \\ Nanoengineering, University of California San Diego, 3855 Health Sciences \\ Drive, La Jolla, CA 92093-0815, USA. ${ }^{3}$ Department of Bioengineering, \\ University of California San Diego, 3855 Health Sciences Drive, La Jolla, CA \\ 92093-0815, USA. ${ }^{4}$ Division of Molecular Virology, Department of Clinical and \\ Experimental Medicine, Sandbäcksgatan 7, Linköping University, 58183 \\ Linköping, Sweden. ${ }^{5}$ Inception Sciences, 5871 Oberlin Drive, San Diego, CA \\ 92121, USA.
}

Received: 30 May 2014 Accepted: 13 March 2015

Published online: 31 March 2015

\section{References}

1. Fournier P, Schirrmacher V. Randomized clinical studies of anti-tumor vaccination: state of the art in 2008. Expert Rev Vaccines. 2009;8:51-66.

2. Gattinoni L, Powell Jr DJ, Rosenberg SA, Restifo NP. Adoptive immunotherapy for cancer: building on success. Nat Rev Immunol. 2006;6:383-93.

3. Network TCGA. Comprehensive molecular portraits of human breast tumours. Nature. 2012;490:61-70.

4. Audran R, Peter K, Dannull J, Men Y, Scandella E, Groettrup M, et al. Encapsulation of peptides in biodegradable microspheres prolongs their MHC class-I presentation by dendritic cells and macrophages in vitro. Vaccine. 2003;21:1250-5.

5. Uto T, Wang X, Sato K, Haraguchi M, Akagi T, Akashi M, et al. Targeting of antigen to dendritic cells with poly(gamma-glutamic acid) nanoparticles induces antigen-specific humoral and cellular immunity. J Immunol. 2007;178:2979-86.
6. Diwan M, Tafaghodi M, Samuel J. Enhancement of immune responses by co-delivery of a CpG oligodeoxynucleotide and tetanus toxoid in biodegradable nanospheres. J Control Release. 2002;85:247-62.

7. Lemoine D, Preat V. Polymeric nanoparticles as delivery system for influenza virus glycoproteins. J Control Release. 1998;54:15-27.

8. Fonseca C, Simoes S, Gaspar R. Paclitaxel-loaded PLGA nanoparticles: preparation, physicochemical characterization and in vitro anti-tumoral activity. J Control Release. 2002;83:273-86.

9. des Rieux A, Fievez V, Garinot M, Schneider YJ, Preat V. Nanoparticles as potential oral delivery systems of proteins and vaccines: a mechanistic approach. J Control Release. 2006; 116:1-27.

10. Lee SY, Oh JH, Kim JC, Kim YH, Kim SH, Choi JW. In vivo conjunctival reconstruction using modified PLGA grafts for decreased scar formation and contraction. Biomaterials. 2003;24:5049-59.

11. Ayalasomayajula SP, Kompella UB. Subconjunctivally administered celecoxibPLGA microparticles sustain retinal drug levels and alleviate diabetesinduced oxidative stress in a rat model. Eur J Pharmacol. 2005;511:191-8.

12. Banchereau J, Steinman RM. Dendritic cells and the control of immunity. Nature. 1998;392:245-52.

13. Telusma G, Datta S, Mihajlov I, Ma W, Li J, Yang H, et al. Dendritic cell activating peptides induce distinct cytokine profiles. Int Immunol. 2006;18:1563-73.

14. Saenz R, Souza CD, Huang CT, Larsson M, Esener S, Messmer D. HMGB1derived peptide acts as adjuvant inducing immune responses to peptide and protein antigen. Vaccine. 2010;28:7556-62.

15. Clawson C, Huang CT, Futalan D, Seible DM, Saenz R, Larsson M, et al. Delivery of a peptide via poly(D, L-lactic-co-glycolic) acid nanoparticles enhances its dendritic cell-stimulatory capacity. Nanomedicine. 2010;6:651-61.

16. Song H, Shahverdi K, Huso DL, Wang Y, Fox JJ, Hobbs RF, et al. An immunotolerant HER-2/neu transgenic mouse model of metastatic breast cancer. Clin Cancer Res. 2008;14:6116-24.

17. Inaba K, Inaba M, Romani N, Aya H, Deguchi M, Ikehara S, et al. Generation of large numbers of dendritic cells from mouse bone marrow cultures supplemented with granulocyte/macrophage colony-stimulating factor. J Exp Med. 1992;176:1693-702.

18. Messmer D, Yang H, Telusma G, Knoll F, Li J, Messmer B, et al. High mobility group box protein 1: an endogenous signal for dendritic cell maturation and Th1 polarization. J Immunol. 2004;173:307-13.

19. Uto T, Akagi T, Yoshinaga K, Toyama M, Akashi M, Baba M. The induction of innate and adaptive immunity by biodegradable poly(gamma-glutamic acid) nanoparticles via a TLR4 and MyD88 signaling pathway. Biomaterials. 2011;32:5206-12.

\section{Submit your next manuscript to BioMed Central and take full advantage of:}

- Convenient online submission

- Thorough peer review

- No space constraints or color figure charges

- Immediate publication on acceptance

- Inclusion in PubMed, CAS, Scopus and Google Scholar

- Research which is freely available for redistribution 